\title{
High-multiplicity neutron events registered by NEMESIS experiment
}

\section{Kasztelan, ${ }^{a, *}$ T. Enqvist, ${ }^{b}$ K. Jędrzejczak, ${ }^{a}$ J. Joutsenvaara, ${ }^{d}$ O. Kotavaara, ${ }^{d}$ P. Kuusiniemi, ${ }^{b}$ K.K. Loo, ${ }^{f}$ J. Orzechowski, ${ }^{a}$ J. Puputti, ${ }^{d}$ A. Sobkow, ${ }^{a}$ M. Słupecki, ${ }^{e}$ J. Szabelski, ${ }^{a}$ I. Usoskin, ${ }^{c}$ W.H. Trzaska ${ }^{b}$ and T.E. Ward ${ }^{g, h}$}

${ }^{a}$ National Centre for Nuclear Research, 28 Pułku Strzelców Kaniowskich 69, Łódź, Poland

${ }^{b}$ Department of Physics, University of Jyväskylä, P.O. Box 35, FI-40014 University of Jyväskylä, Finland

${ }^{c}$ University of Oulu, Sodankyla Geophysical Observatory, P.O. Box 3000, FIN-99600 Sodankyla, Finland

${ }^{d}$ University of Oulu, Kerttu Saalasti Institute, Pajatie 5, 85500 Nivala, Finland

${ }^{e}$ Helsinki Institute of Physics (HIP), P.O. Box 64, 00014 University of Helsinki, Finland

${ }^{f}$ Institut für Physik (IPH), Johannes Gutenberg-Universität Mainz(JGU), Staudingerweg 7, 55128 Mainz,Germany

${ }^{g}$ High Energy Physics (HEP), U.S. Department of Energy, SC-25/Germantown Building, 1000 Independence Ave., SW, Washington, D.C., 20585, United States

${ }^{h}$ TechSource, Santa Fe, NM, United States

E-mail: marcin.kasztelan@ncbj.gov.pl

\begin{abstract}
Neutron-induced interactions contribute to the signal-mimicking background in deep-underground searches for exotic phenomena such as Dark Matter, neutrino-less double beta decay, proton decay, etc. Apart from radioactive decay, the primary source of neutrons underground are high-energy muons from cosmic showers. While the maximum number of fission neutrons is around six and energies around one $\mathrm{MeV}$, muon-induced interactions may generate hundreds of neutrons, also with high energies. Furthermore, these processes are not yet reproduced in numerical simulations with sufficient reliability. The main goal of the NEMESIS experiment is to improve our knowledge and understanding of cosmic muon-induced neutron production in high- $\mathrm{Z}$ targets. NEMESIS (New Emma MEasurementS Including neutronS) is taking data at a depth of 210 m.w.e. in Callio Lab in the Pyhäsalmi mine, Finland.
\end{abstract}

$37^{\text {th }}$ International Cosmic Ray Conference (ICRC 2021)

July 12th-23rd, 2021

Online - Berlin, Germany

\footnotetext{
*Presenter
} 


\section{Introduction}

The neutron flux in the vicinity of underground experiments is important for the estimation of the background for different studies. Contributing neutrons emerge from different fission reactions in surrounding rock etc., and from neutron-induced interactions. A fission reaction produces about six neutrons with energies around one MeV. Muon-induced interactions may generate hundreds of neutrons and with higher energies. Results of that process is not well reproduced in computer simulations. The main goal of the NEMESIS experiment is to measure multi-neutron events occurring in muon interactions in different targets. In this work results obtained from lead target are presented. NEMESIS (New Emma MEasurementS Including neutronS) is taking data at a depth of 210 m.w.e. (75m) in Callio Lab [1] at the Pyhäsalmi mine [2] in Finland since November 2019.

The neutron setup consists of fourteen ${ }^{3} \mathrm{He}$ counters placed in polyethylene blocks. Data from the helium counters and muon scintillation detectors are collected by frontend electronics digitizing waveforms signals. Waveforms are long enough to include muons and delayed neutron signals from helium counters. The preliminary neutron multiplicity spectra shown here include a 349-day (live time) run with a $565 \mathrm{~kg} \mathrm{~Pb}$ target, a 166-day run without the target, and the outcome of the relevant Geant4 simulations.

\section{The first NEMESIS setup}

The first NEMESIS setup is shown schematically in Fig.1. Fig.2 is a photo of the central part only. The first NEMESIS setup is a demonstrator of the full-size NEMESIS Dark Matter experiment and uses the EMMA infrastructure [3] together with the Roland Maze outreach project detectors [4]. This solution allows for the combination of muon tracking with the position-sensitive registration of neutrons. This gives us a muon-induced neutron spectra, yielding muon-induced neutron spectra, neutron production yields, and lateral distributions for the observed high-multiplicity events (in one dimension).

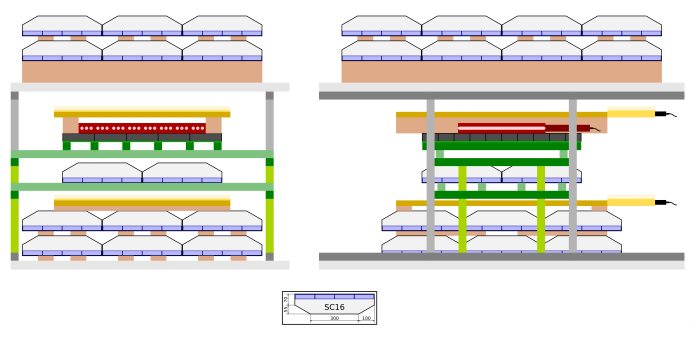

Figure 1: NEMESIS front and side view. There are 5 layers of $\mathrm{SC} 16$ detectors (blue) and a $5 \mathrm{~cm} \mathrm{~Pb}$ target in the center (dark gray). The $14{ }^{3} \mathrm{He}$ neutron counters are polyethylene blocks on the top of $\mathrm{Pb}$. The yellow layers represent 2 scintillation detectors, $1 m^{2}$ each.

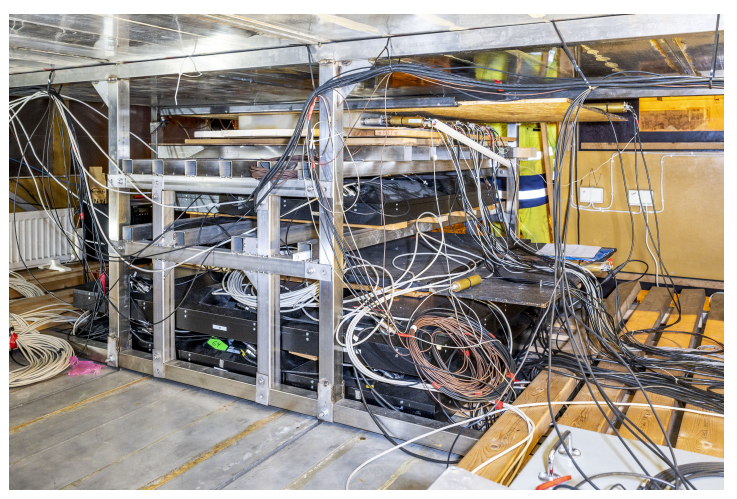

Figure 2: Photo of the central part of the first NEMESIS setup.

The main element of the setup is the array of fourteen proportional counters [5], filled with ${ }^{3} \mathrm{He}$ at $4 \mathrm{~atm}$, and surrounded by polyethylene (PE) $75 \times 6.4 \times 55 \mathrm{~cm}^{3}, \sim 25 \mathrm{~kg}$. PE block acts as 
a moderator slowing-down neutrons and increasing their capture probability. The helium counters were produced by $\mathrm{ZdAJ}[6]$ and are 1 " in diameter and $50 \mathrm{~cm}$ in length.

NEMESIS helium counters have been previously used for neutron background measurements in several European underground laboratories [7-9] as part of the ILIAS and BSUIN projects [10]. A custom-made neutron acquisition system (NDAQ) collects data from ${ }^{3} \mathrm{He}$ detectors and two $1 \mathrm{~m}^{2}$ amplitude-sensitive scintillation detectors.

The muon-tracking part of the setup (Fig.1) consist of 46 SC16 [3] scintillator EMMA modules forming a 5-layer, 736-pixel muon telescope. The SC16 modules were designed for an extended use underground. They were used, for instance, to measure muon flux at LSC in Canfranc, Spain [11]. Each SC16 has a $50 \times 50 \mathrm{~cm}^{2}$ footprint and consists of 16 individual pixels $(12.5 \times 12.5 \times$ $3.0 \mathrm{~cm}^{3}$ ) read by a SiPM with optical fiber. Each module has one common time and one hitpattern output [12]. These pixelized detectors worked in hodoscopic mode. Data from the muon telescope are collected by a separate data acquisition system (EMMA DAQ) requiring offline clock synchronization procedure with NDAQ. EMMA DAQ is triggered when at least one of two top layers and at least one from bottom layers of SC16 have signals in coincidence.

Neutron detectors generate the main NEMESIS trigger. The NDAQ frontend electronic modules distribute the trigger to all channels via a common bus. Each helium counter triggers all the others and the two Maze Project scintillators. All signals are recorded by NDAQ. Data are collected as waveforms for further offline analysis. For each event, a clock synchronization signal is send to EMMA DAQ. NDAQ consist of a 8-bit analogue-to-digital converters (ADC) coupled to circular memory

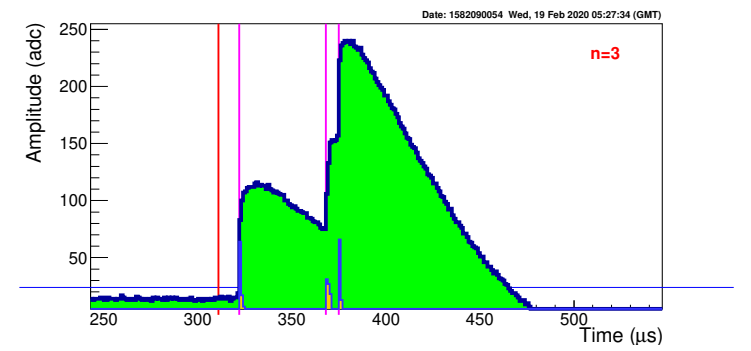

Figure 3: Example of multiple neutron signal registered in single ${ }^{3} \mathrm{He}$ counter with identified 3 neutrons. Only fraction of whole registered wave-form is shown to visualize the structure of neutron signals. buffer with $2 \mathrm{k}$ ADC samples. A signal sampling frequency of $1 \mathrm{MHz}$ is appropriate for the current detection requirements. Data are transferred via a USB to the local PC, where they are stored as ROOT files [15]. To increase the dynamic range, each $1 \mathrm{~m}^{2}$ scintillator detector provides two waveform signals, one from PMT's anode, and $\sim 30$ times weaker signal from the $6^{\text {th }}$ dynode. The waveforms are stored by the frontend electronics (FE) in $2 \mathrm{~ms}$ circular buffers. The first $350 \mu \mathrm{s}$ precede the neutron trigger to capture the prompt muon signal in the scintillation detectors, accounting for neutron thermalization time. Neutrons use on average about $50 \mu s$ for thermalization before their registration. The rest of waveform may include signals from subsequent neutrons, arriving with a delay caused by the time spread of the thermalization process.

The first NEMESIS run lasted 349 days. The $565 \mathrm{~kg} \mathrm{~Pb}$ target consisted of fifty standard $\mathrm{Pb}$ bricks, $20 \times 10 \times 5 \mathrm{~cm}^{3}$ each, forming a $1 \mathrm{~m}^{2}, 5 \mathrm{~cm}$ thick, uniform layer. Subsequently, the lead was removed for 7-month background measurement. From August, we intend to run with a copper target.

In high-multiplicity events, the neutron signals may overlap, making them difficult to distinguish and count correctly, resulting in efficiency loss. The example in Fig.3 is relatively easy to interpret. However, overflow signals are often encountered with large neutron multiplicities. We are improving 
the detection method for the NEMESIS upgrade, including better software, higher sampling rate, higher dynamics of amplitude measurements, and additional helium counters. Also considered are improvements in muon tracking and passive shield against neutron flux from the surrounding rock. These measures will increase the registration efficiency and lower the background.

\section{Research methodology}

Following a neutron capture by a ${ }^{3} \mathrm{He}$ nucleus (eq.1), the reaction products are emitted in opposite directions. Due to negligible kinetic energy of the primary neutron, there is no need to correct for the centre of mass velocity. The so called "wall-effect" [5], visible in the amplitude spectrum (Fig.6), appears when either the proton or tritium produced in the capture reaction escapes from the active volume of the counter before depositing their total energy. For non relativistic neutrons $\left(E_{n}<100 \mathrm{keV}\right)$, capture cross section [13] is inversely proportional to the neutron velocity. For neutron witch energies from $10^{-5}$ to $10^{4} \mathrm{eV}$ that relation can be parameterized as follows: $\sigma\left(E_{n} /\right.$ barn $)=847 E_{n}^{-0.5}[\mathrm{eV}]$.

Used nuclear reaction to detect neutrons [14]:

$$
n+{ }_{2}^{3} \mathrm{He} \rightarrow{ }_{1}^{3} \mathrm{H}+p+Q(764 k e V)
$$

NEMESIS neutron identification proceeds in stages. All analysis was done offline. First waveform differences between consecutive ADC samples are calculated. This is depicted by the orange inset in Fig.3. The waveforms are scanned for signals exceeding a selected threshold. The corresponding $\mathrm{ADC}$ channel provides a reference for the registration time. The value resulting from adding three consecutive differences is proportional to the amplitude of the measured signal. The second important parameter is the largest difference in the vicinity of the potential signal. This value is proportional to the rise time of the registered pulse. The rise time was used for selecting neutron signals, as shown in Fig.5.

Data collected by our USB interface required clustering. Then, a search for potential signals in the waveform was performed. Next, neutron signals were identified through cuts on the amplitude and maximum derivative between the consecutive ADC samples. The procedure is illustrated in Fig.5. Proper neutron signals are inside the triangular area delimited by the cut lines.

The selection procedure is carried out for each counter separately after selecting the appropriate cuts presented in Fig. 5. Cutting lines have different tasks. The red line eliminates low amplitude signals caused by electronic noise and contributions from the large amount of gamma photons recorded in the counter (in some cases). The blue line rejects signals with higher amplitudes which are come from alpha particles of the helium counter's own radioactivity. Our own radioactivity of helium counters is at the level $0.78 \mathrm{MeV}^{-1} \mathrm{~h}^{-1}$. These alpha particles are emitted inwards the counter from the housing material as a result of decays from natural radioactivity chains. The green line eliminates potential signals from the high voltage leakage in the HV-BNC connector between the detector and the frontend electronics.

Fig. 6 shows the amplitude distributions recorded in a single ${ }^{3} \mathrm{He}$ counter after applying the selection method described earlier. The obtained amplitude distribution (green line) perfectly corresponds to the model of this type of neutron counters [5]. The peak with an ADC value of 100 corresponds to the full energy peak $(764 \mathrm{keV})$ of the reaction (eq. 1). 


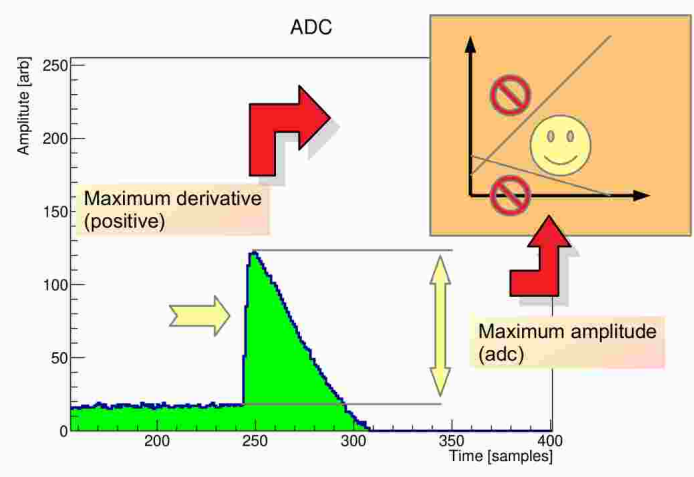

Figure 4: The idea of creating parameters on the basis of which cuts will be made to select signals from neutrons. Amplitude value signal and the maximum difference between successive samples for each pulse is marked in Fig.5

\section{Measurement results}

The outcome of the NEMESIS measurement is summarised in Fig.7. The ambient neutron background dominates multiplicityone events. The results with and without the $\mathrm{Pb}$ target differ just by $17 \%$. For multiplicity two, background events are already suppressed by order of magnitude. The neutron spectra for multiplicity two and above show a distinct linear dependence in the log-log scale, indicating a simple power-law dependence. The fits yielded $-3.15 \pm 0.03$ for the slope of the $\mathrm{Pb}$ run and $-6.92 \pm 2.29$ for the background run. For multiplicities around and above 20, the measured points fall short of the extrapolated line. This was expected, as no efficiency corrections were applied. This will be done after the completion of the simulations campaign to characterise the performance of the experimental setup.

The largest registered event had 33 identified neutrons. For that event, correcting for nearly $8 \%$ detection efficiency, determined with Geant 4 , the required neutron emission is around 400 neutrons. The origin of such a large multiplicity of neutrons is still not known.

Aspects related to possible Dark Matter annihilation signatures in NEMESIS neutron multiplicity spectra are discussed in ICRC 2021 contribution \#394 and published in the proceedings of 
Neutron multiplicity comparison

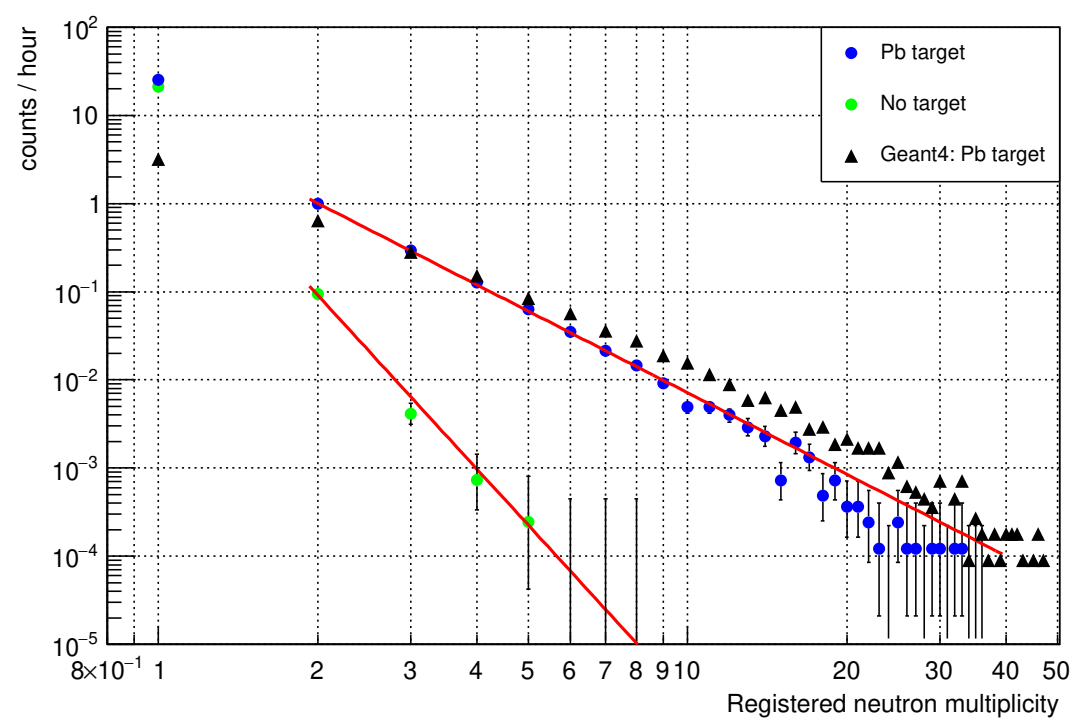

Figure 7: Neutron multiplicities recorded with the lead target (blue dots), and without a target (green dots). Black triangles show Geant 4 predictions.

this conference [16].

\section{Geant4 simulations}

We also performed Monte Carlo simulations using Geant4 [17] version 10.06 .02 with QGSP_BERT_HP Physics List with thermal scattering model $S(\alpha, \beta)$ [18]. This model, important for neutrons with energies below $4 \mathrm{eV}$, considers chemically bound atoms in materials like polyethylene or water. The neutron data files for High Precision Neutron models are based on ENDF files.

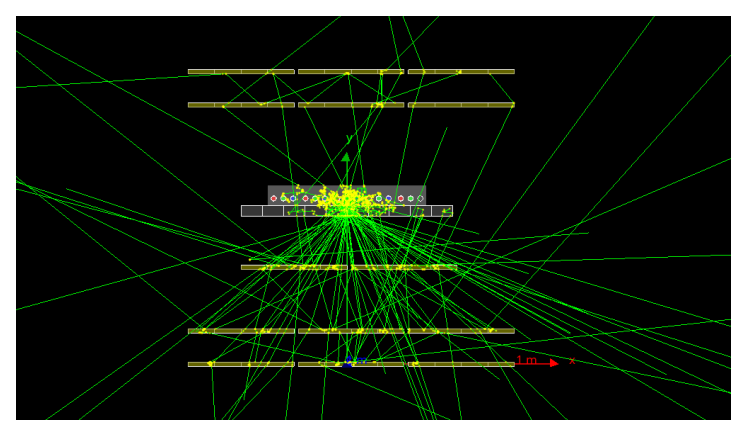

Figure 8: A sample of Geant 4 neutron tracks. All of the simulated neutrons had $10 \mathrm{MeV}$ initial energy and were emitted from the center of the $\mathrm{Pb}$ target.

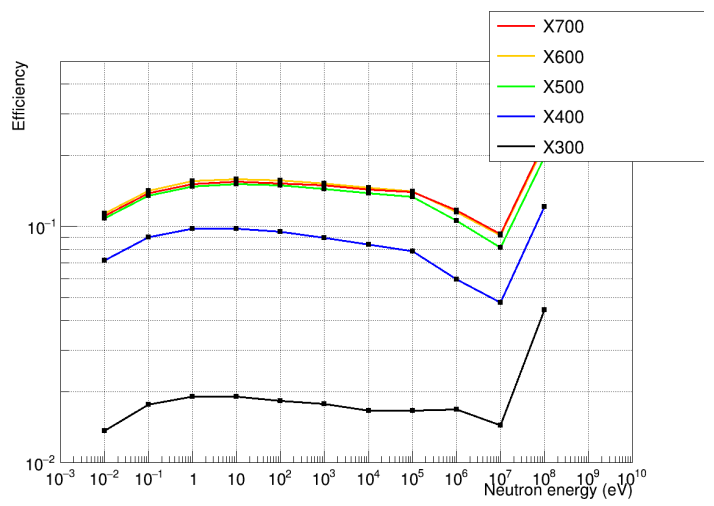

Figure 9: Neutron registration efficiency as a function of energy, calculated for different source positions. X700 is the most central and X300 is the most peripheral location. 
We have performed detection efficiency simulations for a broad range of neutron energies (Fig. 8). We expect similarity with spallation spectra with the average neutron energy around 2 $\mathrm{MeV}$. Neutron registration efficiency, summed up for all counters, reaches $16 \%$ for neutron energies around $10 \mathrm{eV}$, and $10 \%$ for neutrons in the $1 \div 10 \mathrm{MeV}$ range (see Fig. 9). For neutrons with energies above $10 \mathrm{MeV}$, the production of secondary neutrons from the $\mathrm{Pb}(\mathrm{n}, \mathrm{xn})$ reaction starts to play a dominant role.

Assuming muon distribution at sea-level from Guan et al. [19], based on the standard Gaisser's formula, and a simplified lepton propagation through matter with Muon Monte Carlo (MMC) code [20], we have simulated 50 million CR muons hitting the NEMESIS Pb target. The outcome was normalised to the well-known muon flux value of $1.29 \pm 0.06 \mathrm{~m}^{-2} \mathrm{~s}^{-1}$, measured previously by EMMA [3]. The best match with the measured neutron spectrum, shown in Fig.7, was obtained assuming a constant $6.6 \%$ efficiency for our setup. Because of the included simplifications, this value represents the lower limit of NEMESIS neutron efficiency. Other preliminary estimates yielded an $8 \pm 2 \%$ efficiency.

The presented results are preliminary. Further analysis and simulations are ongoing. We should be able to present them at the next ICRC conference.

\section{Conclusion}

After the first 18 months of operation, NEMESIS delivered valuable data on muon-induced neutron multiplicities and yields in lead. Data on multiplicities and yield from copper will follow. When properly analysed, the results will impact simulation software and improve the understanding of the neutron background in underground experiments.

The NEMESIS setup is upgradeable. As the next step, we intend to double the number of neutron detectors and increase target mass. There are plans to move simulation to the local computer clusters.

\section{Acknowledgements}

This work was financially supported in part by the EU (INTERREG for Baltic Sea programme) as part of the BSUIN project, and by the Polish Ministry of Science and Higher Education (grant no. 3988/INTERREG BSR/2018/2).

\section{References}

[1] Callio webpage: https://callio.info. Accessed: 2021-06-22

[2] W. H. Trzaska et al., Possibilities for Underground Physics in the Pyhäsalmi mine, 2018, arXiv: 1810.00909 .

[3] P. Kuusiniemi et al., Performance of tracking stations of the underground cosmic-ray detector array EMMA. Astropart. Phys., 102,67-76, 2018.

[4] M. Kasztelan et al., Detector Calibration and Data Acquisition System in the Roland Maze Project, 2006. ECRS 2006 Proceedings. 
[5] Z. Dębicki at al., Helium counters for low neutron flux measurements, Astrophys. Space Sci. Trans., 7, 511-514, 2011

[6] ZdAJ-HITEC webpage: http://hitecpoland.eu

[7] Z. Debicki et al., Thermal neutrons at Gran Sasso. Nucl. Phys. B Proc. Suppl., 196, 429-432, 2009

[8] Z. Debicki at al., Neutron flux measurements in the Gran Sasso national laboratory and in the Slanic Prahova Salt Mine, Nucl. Instrum. Meth. A, 910, 133-138, 2018

[9] Kinga Polaczek-Grelik at al, Natural background radiation at Lab 2 of Callio Lab, Pyhäsalmi mine in Finland, Nucl. Instrum. Meth. A, 969, 164015, 2020

[10] BSUIN webpage: http://bsuin.eu/ Accessed: 2021-06-28

[11] W. H. Trzaska et al. Cosmic-ray muon flux at Canfranc Underground Laboratory. Eur. Phys. J. C, 79(8):721, 2019, 1902.00868.

[12] E. V. Akhrameev et al. Multi-pixel Geiger-mode avalanche photodiode and wavelength shifting fibre readout of plastic scintillator counters of the EMMA underground experiment. Nucl. Instrum. Meth. A, 610, 419-422, 2009, 0901.4675

[13] D.A. Brown et al. ENDF/B-VIII.0: The 8th Major Release of the Nuclear Reaction Data Library with CIELO-project Cross Sections, New Standards and Thermal Scattering Data, Nuclear Data Sheets, 148, 2018, 1-142, https://doi.org/10.1016/j.nds.2018.02.001.

[14] Knoll, Glenn F., Radiation Detection and Measurement, John Wiley and Sons, New York, 2000

[15] Brun, R. and Rademakers, F., ROOT: An object oriented data analysis framework, doi: "10.1016/S0168-9002(97)00048-X", Nucl. Instrum. Meth. A, Vol. 389, 81-86, 1997

[16] W.H. Trzaska et al. New NEMESIS Results, 2021. ICRC 2021 Proceedings.

[17] Recent developments in Geant4, Nucl. Instrum. Meth. A, 835, 2016, 186-225, ISSN 0168-9002

[18] C.T. Ballinger. The direct S(alpha,beta) method for thermal nuetron scattering. American Nuclear Society, Inc., La Grange Park, IL (United States), Dec 1995.

[19] M. Guan et al., A parametrization of the cosmic-ray muon flux at sea-level, https://arxiv.org/abs/1509.06176v1

[20] D. Chirkin et al., Propagating leptons through matter with Muon Monte Carlo (MMC). https://arxiv.org/abs/hep-ph/0407075 